\title{
Stent-Retriever Thrombectomy: Impact on the Future of Interventional Stroke Treatment
}

\author{
P. Mordasini · C. Zubler · K. Wha-Vei Hsieh • \\ P. K. Chan · J. Gralla
}

Received: 13 December 2013 / Accepted: 12 February 2014 / Published online: 22 February 2014

(C) Springer-Verlag Berlin Heidelberg 2014

\begin{abstract}
Endovascular treatment for acute ischemic stroke has evolved in the past years. The current development of stent-retriever thrombectomy is a landmark in the clinical treatment and study results of acute ischemic stroke. This review summarizes the recent study results, elucidates the shortcomings of endovascular stroke treatment, and takes the opportunity for an outlook on the role of stroke interventions in the future.
\end{abstract}

Keywords Endovascular stroke treatment $\cdot$ Mechanical thrombectomy $\cdot$ Stent-retriever

\section{Introduction}

Acute ischemic stroke is one of the major causes of morbidity and mortality in Western countries. The neurological outcome depends on the length of time between onset of symptoms and treatment, and on whether recanalization of the occluded vessel can be achieved. The chance of an independent life after stroke increases 4.4 times for patients with successful recanalization compared with patients without recanalization. At the same time, mortality rate decreases fourfold [1].

P. Mordasini, MD, MSc $(\varangle)$

Department of Interventional and Diagnostic Neuroradiology,

University of Bern, Bern, Switzerland

e-mail: pasquale.mordasini@insel.ch

C. Zubler, MD · K. Wha-Vei Hsieh, MD · J. Gralla, MD, MSc Department of Interventional and Diagnostic Neuroradiology, University of Bern, Bern, Switzerland

P. K. Chan, MD

Department of Radiology, Kuala Lumpur Hospital,

Kuala Lumpur, Malaysia
The prevalence of acute ischemic stroke is age dependent and increases significantly above the age of 65 years [2]. Taking into account the expected demographic development in the aging population of industrialized nations, the total number of strokes is likely to increase within the next decades. Based on the demographic data from Germany, the number of inhabitants above the age of 65 years, and therefore within the age more prone for ischemic cerebrovascular events, rose from approximately 7 million $(10 \%$ of the overall population) in 1950 to 17 million (23\%) in 2013 and is expected to rise to approximately 23 million (33\%) in 2050 (Fig. 1). Optimized and effective treatment approaches for this devastating disease are mandatory in the future. Intravenously administered tissue plasminogen activator (IV tPA) has proven to be effective in the treatment of stroke patients when applied within a 4.5-h time window.

However, the success of recanalization depends on the occlusion site; proximal occlusions of large vessels such as the internal carotid artery (ICA) have a poor recanalization rate after either IV tPA or IAT [3, 4]. Furthermore, the time window for treatment and the recanalization rate is limited [3-5], and the application of thrombolytic drugs increases the risk of symptomatic intracranial hemorrhage (sICH) [6]. Recent studies have examined whether endovascular recanalization techniques can accelerate the process of recanalization, increase the recanalization rate, and even expand the window of opportunity. However, endovascular stroke treatment has failed to show superiority to sole intravenous thrombolysis in the recently published three randomized controlled trials (RCTs): Interventional Management of Stroke (IMS)-III, SYNTHESIS Expansion, and MR RESCUE [7-9].

This review describes the recent evolution of endovascular stroke treatment and summarizes the clinical results of stent-retriever (SR) thrombectomy. On the basis of the cur- 


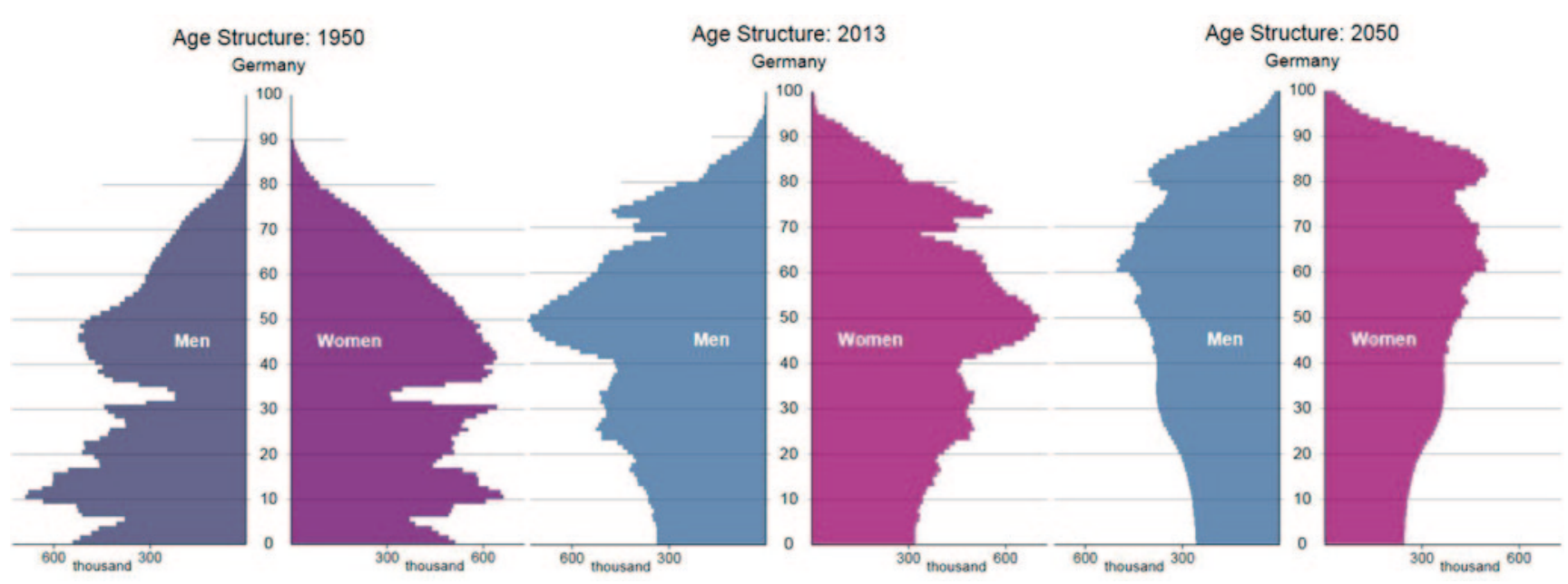

Fig. 1 Demographic development of the German population from 1950 to 2013 and estimated development until 2050. The number of people above the age of 65 years is increasing relatively and absolutely in the total population (Statistisches Bundesamt 2009).

rent data on stroke treatment, the authors advocate the role of the endovascular approaches in the future.

\section{Thrombectomy Techniques}

The various techniques and approaches for mechanical thrombectomy (MT) have evolved over time and can be divided into three categories: proximal aspiration, distal thrombectomy and in-thrombus approaches such as placement of selfexpandable stents, and SR thrombectomy (Fig. 2). All MT

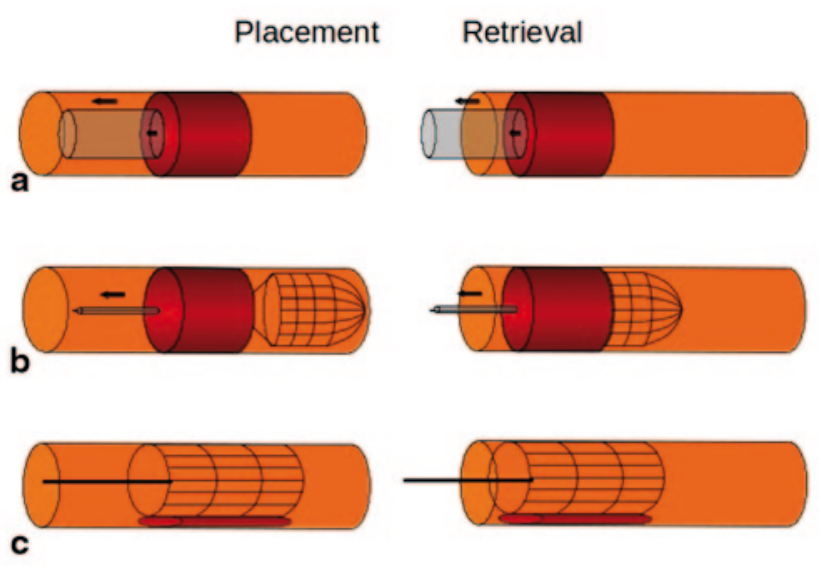

Fig. 2 a Proximal thrombectomy by aspiration. A large-diameter catheter is advanced to the proximal surface of the thrombus. Under continuous aspiration, the device is then retrieved, mobilizing the thrombus from the cranial vessels. b Distal thrombectomy. Passing the occlusion site and developing a cage-, net-, or coil-like device distally to the thrombus, the device is retrieved together with the thrombus. c Stent-retriever thrombectomy. After placement of the self-expandable device and thrombus compression, generating a temporal bypass effect, the device is retrieved together with the thrombus from the intracranial vasculature devices are delivered by endovascular access to the occlusion site. The various devices can be divided into three major groups according to where they apply force on the thrombus.

Proximal devices apply force to the proximal base of the thrombus; this group includes various aspiration catheters and systems. Distal devices approach the thrombus proximally, but then are advanced by a microcatheter to pass the thrombus and to be unsheathed behind it, where force is applied to the distal base of the thrombus; this group includes brush-, basket-, or coil-like devices. Proximal and distal approaches can be considered as first-generation approaches. The second-generation devices include stent-like devices that are placed across the occlusion side, deployed within the thrombus and then retrieved; this group includes various self-expandable stent-retrievers (SR).

\section{Studies on First-Generation Devices}

First-generation devices have been studied extensively in single-arm studies. The Penumbra system (Penumbra, Alameda, CA, USA) is a refinement of the proximal thrombectomy technique and applies continuous aspiration in conjunction with mechanical fragmentation.

The Penumbra Pivotal Stroke Trial [10] prospectively examined the results in 125 stroke patients (mean National Institute of Health Stroke Scale (NIHSS) score: 18) within a window of $8 \mathrm{~h}$ after onset of symptoms. Recanalization of the target vessel was achieved in $81.6 \%$ of patients. Despite the convincing recanalization rate, clinical outcome was poor; favorable clinical outcome was achieved in only $25 \%$ of all patients and in $29 \%$ of those with successful recanalization. The rate of sICH was $11.2 \%$, and overall mortality was $32.8 \%$. Serious adverse events occurred in $3.2 \%$ of cases. The discrepancy between high recanalization rates and poor 
clinical outcome has been discussed intensively. A possible explanation is due to the grading system of TIMI used in the study. A full recanalization could be achieved independent of reperfusion of the brain, an aspect which led to the application of the TICI grading in future studies thereafter.

The initial Mechanical Embolus Removal in Cerebral Ischemia (MERCI) trial evaluated the safety and efficacy of the Merci system in the setting of acute stroke within $8 \mathrm{~h}$ of onset in 151 patients (mean NIHSS score: 20) who were ineligible for intra-arterial thrombolysis (IAT) [11]. The trial included patients with anterior circulation $(90 \%)$ and posterior circulation $(10 \%)$ stroke. Successful recanalization was achieved in $46 \%$ of the treated patients, significantly higher compared with the subgroup, with a spontaneous recanalization rate of $18 \%$ in the Prolyse in Acute Cerebral Thromboembolism (PROACT) II trial [12]. Favorable clinical outcome was achieved in $27.7 \%$ of patients. The mean procedure duration was $2.1 \mathrm{~h}$; clinically significant procedural complications occurred in $7.1 \%$ of patients, and the sICH rate was $7.8 \%$.

The Multi-MERCI trial was an international, single-arm trial that investigated 164 patients (mean NIHSS score: 19) within an 8-h window after symptom onset [13]. The primary end point was target vessel recanalization. In contrast to the MERCI trial, prior treatment with IV tPA, IAT, or other mechanical techniques was allowed, and new generations of the Merci device were included. Successful recanalization was achieved in $57.3 \%$ of cases using the retriever alone and $69.5 \%$ in conjunction with other treatment modalities. Favorable clinical outcome was achieved in $36 \%$ of patients. Mean procedure duration was $1.6 \mathrm{~h}$ and, therefore, again remarkably long for a mechanical approach. Clinically significant procedural complications occurred in $5.5 \%$ of patients, and the sICH rate was $9.8 \%$.

The introduction of aspiration and distal devices has been a landmark of mechanical recanalization in stroke treatment, but the studies illustrated high recanalization rates in correlation to moderate or poor clinical results. However, due to novel developments in aspiration catheter technology, this technique might show improved results in the future. Due to their early Food and Drug Administration approval, both approaches have been assessed within the IMS-III trial [7].

\section{Second-Generation Devices}

The most recently introduced mechanical devices for acute stroke treatment are self-expandable, stent-like thrombectomy devices. Combining the advantages of temporary stenting with immediate flow restoration without the need for permanent implantation plus thrombectomy with definitive thrombus removal, stent-retrieval devices offer a promising new treatment option for acute ischemic stroke. Numerous variants of this device type are currently under development or in first clinical trials (e.g., Solitaire/Covidien, TREVO/Concentric Medical, ERIC/MicroVention, 3D Separator/Penumbra, Preset/Phenox etc.).

Castano et al. [14] reported in an initial study in 2010 on their experience in 20 acute stroke patients within an 8-h time window. Successful recanalization was achieved in $90 \%$ of cases with a mean procedural time of $50 \mathrm{~min}$. A favorable clinical outcome was attained in $45 \%$, and sICH rate was $10 \%$. These results have been reproduced by various small case series and retrospective studies using SRs, which have shown similar successful recanalization rates $(85-91 \%)$ and fast procedural times (40-55 $\mathrm{min})$ with comparable rates of favorable clinical outcome (42-55\%) [15-18].

The largest prospective study on SR thrombectomy was the Solitaire FR Thrombectomy for Acute Revascularization (STAR) trial [19]. This prospective, international, single-arm study enrolled 202 consecutive patients (mean age: 72 years, mean baseline NIHSS score: 17) with anterior circulation occlusion treated within an 8-h time window. For this study, all patients were selected on the basis of proximal vessel occlusion in cross-sectional imaging; furthermore, the use of a balloon guide catheter and flow reversal during thrombectomy was mandatory. The primary outcome, recanalization of Thrombolysis in Cerebral Infarction (TICI) $\geq 2 b$, was core laboratory controlled and achieved in $79.2 \%$ of cases, resulting in a favorable clinical outcome modified Ranking Scale (mRS) 0-2 in 57.9\%. Mean procedural time from placement of balloon guide catheter to recanalization was $29 \mathrm{~min}$. Mortality was $6.9 \%$, sICH occurred in $1.5 \%$.

Unlike previous studies with first-generation devices, studies of SR thrombectomy revealed high recanalization rates, fast procedural times, and low rates of $\mathrm{sICH}$, which ultimately correlate with the high rates of favorable clinical outcome (Table 1).

\section{Comparison of First- and Second-Generation Devices}

The Solitaire FR with the Intention for Thrombectomy (SWIFT) trial and the TREVO II trial were randomized trials comparing the efficacy and safety of second-generation devices (Solitaire FR and TREVO) with that of a first-generation device, the Merci device. Both trials illustrated the inferiority of the first-generation devices compared with SRs.

The SWIFT trial [20] randomized 58 patients to the Solitaire group and 55 patients to the Merci group. The primary efficacy outcome, Thrombolysis in Myocardial Infarction (TIMI) 2-3 without sICH, was achieved more often in the Solitaire group (61\%) than in the Merci group (24\%). Fur- 
Table 1 Core laboratory-controlled study results of mechanical thrombectomy using stent-retrievers

\begin{tabular}{|c|c|c|c|c|c|}
\hline & $\begin{array}{l}\text { TREVO } \\
2012\end{array}$ & $\begin{array}{l}\text { TREVO II } \\
\text { (SR group only) } \\
2012 \\
\end{array}$ & $\begin{array}{l}\text { Retrospective study } \\
2012\end{array}$ & $\begin{array}{l}\text { SWIFT } \\
\text { (SR group only) } \\
2012 \\
\end{array}$ & $\begin{array}{l}\text { STAR } \\
2013\end{array}$ \\
\hline Device & TREVO & TREVO & Solitaire & Solitaire & Solitaire \\
\hline Number of patients & 60 & 88 & 141 & 58 & 202 \\
\hline Age (mean) & 65 (median) & 67.4 & 66.3 & 67.1 & 68.4 \\
\hline Baseline NIHSS score (mean) & 18 & 19 & 18 & 18 & 17 \\
\hline Posterior circulation (\%) & 8.3 & 8 & 11 & 1.7 & \\
\hline Successful recanalization (\%) & $78.3(\mathrm{TICI} \geq 2 \mathrm{a})$ & $86(\mathrm{mTICI} \geq 2)$ & $85(\mathrm{TICI} \geq 2 \mathrm{~b})$ & 75.9 (TICI $\geq 2 b)$ & $79.2(\mathrm{TICI} \geq 2 \mathrm{~b})$ \\
\hline $\mathrm{mRS} \leq 2$ at 3 months $(\%)$ & 55.0 & 40 & 55 & 36.4 & 57.9 \\
\hline Mortality at 3 months (\%) & 20 & 33 & 20 & 17.2 & 6.9 \\
\hline sICH at $24 \mathrm{~h}(\%)$ & 5 & 7 & 4 & 1.7 & 1.5 \\
\hline
\end{tabular}

TREVO study [27], TREVO II trial [21], Retrospective study on Solitaire [16], SWIFT trial [20], STAR study [19]

$S R$ stent-retriever, SWIFT Solitaire FR with the Intention for Thrombectomy, STAR Solitaire FR Thrombectomy for Acute Revascularization, sICH symptomatic intracranial hemorrhage, NIHSS National Institute of Stroke Scale, TICI Thrombolysis in Cerebral Infarction, $m$ TICI modified Thrombolysis in Cerebral Infarction, $m R S$ modified Ranking Scale

thermore, good 3-month neurological outcome (defined as $\mathrm{mRS} \leq 2$ or equal to the prestroke $\mathrm{mRS}$ if the prestroke mRS was $>2$ or NIHSS score improvement of 10 points or more) was significantly more often achieved with the Solitaire than with Merci device (58 versus 33\%), while 90-day mortality was higher in the Merci group compared with the Solitaire group (17 versus $38 \%$ ).

The TREVO II trial [21] randomly assigned 88 patients to the TREVO Retriever group and 90 patients to Merci Retriever group. Recanalization rate (TICI 2-3) was significantly higher in the TREVO group (86\%) compared with the Merci group (60\%). Incidence of the primary safety end point did not differ between groups.

\section{The Current Study Situation}

The most recent landmark in stroke research was the publication of three RCTs: IMS-III, SYNTHESIS Expansion, and MR RESCUE [7-9]. All trials compared the clinical outcome of stroke patients randomly assigned to IV tPA or MT in combination with IV tPA. All three trials were unable to show superiority of the endovascular approach compared with the sole application of IV tPA in different study settings. The studies have been extensively discussed in the near past $[22,23]$. Criticism on the study design mainly focuses on low recruitment rates of the studies, inclusion of patients without adequate imaging and proven proximal vessel occlusion, delayed treatment in the endovascular arm, and heterogeneity of the endovascular techniques and protocols. Due to the late clearance of SR, in none of the studies were a substantial number of patients treated with second-generation devices. Taking into account the aforementioned RCT comparing first- and second-generation devices, an impact on the study results can be assumed.

\section{The Shortcoming of Endovascular Treatment}

At the same time, the recent studies and trials revealed major shortcomings of current endovascular stroke treatment approaches, i.e., the missing standardization of the procedures. Although many aspects of acute ischemic stroke are well investigated and the current endovascular approaches show high recanalization rates, impact of patient selection in the peri-procedural management during stroke interventions are poorly understood. In clinical practice as well as in studies, many crucial aspects are undefined or arbitrarily chosen.

The role of peri-procedural anticoagulation and platelet inhibition during stroke interventions is still unknown. Although increased rates of sICH in conjunction with different drugs have been shown by various stroke studies and reviews [24], no common agreement on their application during stroke interventions is established; in fact, the reported protocols differ significantly. This is even more surprising taking into account that many patients are treated by thrombectomy because of pre-existing anticoagulation and contraindication for IV tPA. Furthermore, in case of stent recanalization of access vessels, the role and timing of antiplatelet medication becomes essential.

Various retrospective studies and subgroup analysis have reported different rates of favorable outcome in patients treated endovascularly depending on the anesthesia protocol $[25,26]$. Most data illustrated a significantly worse outcome following general anesthesia (GA) compared with conscious sedation or treatment under local anesthesia of the groin only. However, these studies lack randomization and are biased by the fact that by trend, patients with more complex occlusions (e.g., tandem occlusions) and those restless along with high NIHSS scores and presenting incompliance are treated under GA. Nevertheless, various factors have been considered to contribute to worse outcome after GA. The intubation is time-consuming and causes a clini- 
cally relevant time delay in comparison with patients treated without it. Furthermore, introduction of GA is often accompanied by a decreased systemic blood pressure, resulting in reduced collateral pial blood flow [25]. This reduction of collateral flow might cause infarct growth during intervention until reperfusion is achieved.

Since the introduction of MT, studies illustrated a shift toward peri-procedural complications, some of them with possible impact on patients' outcome (e.g., dissections, perforations, and thromboembolic events into previously unaffected vascular territories) $[10,11,13,19]$. To date, no standardized reporting of peri-interventional complications has been established clinically or in study protocols. For second-generation SR thrombectomy, proximal flow reversal has been advocated and reasonable; however, so far, its application is not mandatory.

\section{An Outlook on Endovascular Stroke Treatment in the Future}

Fast restoration of cerebral blood flow is the principal aim of stroke therapy, associated with better clinical outcome and reduced mortality rates after acute ischemic stroke. Secondgeneration MT devices using SRs have been shown in single-arm studies to achieve high recanalization rates and fast procedural times, which - unlike first-generation devicesultimately correlate with high rates of favorable clinical outcome. The superiority of second-generation devices has been illustrated in trials comparing first- and second-generation devices. Therefore, the recently published RCT on IV tPA versus MT do not reflect the current state of the art in endovascular stroke treatment, as SRs have not been applied in a substantial number of patients in the trials. However, to date, no RCT has proven the superiority of MT in comparison with sole IV tPA.

From the authors' point of view, the current data on endovascular stroke treatment using SRs advocate this approach for standard treatment of acute ischemic stroke in the future. The mechanical approaches have undoubtedly expanded the time window for stroke treatment beyond $4.5 \mathrm{~h}$ and broadened the treatment for patients in whom IV tPA or IAT failed or is contraindicated. Considering the poor recanalization rate and clinical outcome of patients suffering from proximal vessel occlusions and large thrombus burden (e.g., tandem occlusions and ICA termination), SR thrombectomy is likely to become standard treatment also within the 4.5-h time window. RCTs are necessary to confirm this role in the future and to set the protocols of accompanying IV tPA or bridging therapy. The currently ongoing and upcoming trials (e.g., SWIFT Prime, THERAPY, PISTE, THRACE, REVASCAT, THRILL) will elucidate this role and are likely to be positive for MT.
However, although we have a very sufficient tool for fast recanalization in our hands now, interventionists have failed to set up guidelines and standardized protocols for the intervention as well as the peri-procedural patient management yet. Many of these might have a significant impact on patients' outcome and might jeopardize patients' recovery despite successful recanalization. Attention and investigation are urgently required to standardize endovascular stroke treatment for the future.

Conflict of Interest We declare that none of the authors has a conflict of interest. J. Gralla was the Principal Investigator of the STAR study.

\section{References}

1. Rha JH, Saver JL. The impact of recanalization on ischemic stroke outcome: a meta-analysis. Stroke. 2007;38(3):967-73.

2. Seshadri S, Wolf PA. Lifetime risk of stroke and dementia: current concepts, and estimates from the Framingham Study. Lancet Neurol. 2007;6(12):1106-14.

3. Mori E, Yoneda Y, Tabuchi M, Yoshida T, Ohkawa S, Ohsumi Y, et al. Intravenous recombinant tissue plasminogen activator in acute carotid artery territory stroke. Neurology. 1992;42(5):976-82.

4. Saqqur M, Uchino K, Demchuk AM, Molina CA, Garami Z, Calleja $\mathrm{S}$, et al. Site of arterial occlusion identified by transcranial Doppler predicts the response to intravenous thrombolysis for stroke. Stroke. 2007;38(3):948-54.

5. del Zoppo GJ, Poeck K, Pessin MS, Wolpert SM, Furlan AJ, Ferbert A, et al. Recombinant tissue plasminogen activator in acute thrombotic and embolic stroke. Ann Neurol. 1992;32(1):78-86.

6. Brekenfeld C, Remonda L, Nedeltchev K, Arnold M, Mattle HP, Fischer U, et al. Symptomatic intracranial haemorrhage after intraarterial thrombolysis in acute ischaemic stroke: assessment of 294 patients treated with urokinase. J Neurol Neurosurg Psychiatry. 2007;78(3):280-5.

7. Broderick JP, Palesch YY, Demchuk AM, Yeatts SD, Khatri P, Hill $\mathrm{MD}$, et al. Endovascular therapy after intravenous t-PA versus t-PA alone for stroke. N Engl J Med. 2013;368(10):893-903.

8. Ciccone A, Valvassori L, Nichelatti M, Sgoifo A, Ponzio M, Sterzi $\mathrm{R}$, et al. Endovascular treatment for acute ischemic stroke. N Engl J Med. 2013;368(10):904-13.

9. Kidwell CS, Jahan R, Gornbein J, Alger JR, Nenov V, Ajani Z, et al. A trial of imaging selection and endovascular treatment for ischemic stroke. N Engl J Med. 2013;368(10):914-23.

10. The Penumbra Pivotal Stroke Trial Investigators. The penumbra pivotal stroke trial: safety and effectiveness of a new generation of mechanical devices for clot removal in intracranial large vessel occlusive disease. Stroke. 2009;40(8):2761-8.

11. Smith WS, Sung G, Starkman S, Saver JL, Kidwell CS, Gobin YP, et al. Safety and efficacy of mechanical embolectomy in acute ischemic stroke: results of the MERCI trial. Stroke. 2005;36(7):1432-8.

12. Furlan A, Higashida R, Wechsler L, Gent M, Rowley H, Kase C, et al. Intra-arterial prourokinase for acute ischemic stroke. The PROACT II study: a randomized controlled trial. Prolyse in Acute Cerebral Thromboembolism. JAMA. 1999;282(21):2003-11.

13. Smith WS, Sung G, Saver J, Budzik R, Duckwiler G, Liebeskind DS, et al. Mechanical thrombectomy for acute ischemic stroke: final results of the Multi MERCI trial. Stroke. 2008;39(4):1205-12.

14. Castano C, Dorado L, Guerrero C, Millan M, Gomis M, Perez de la Ossa N, et al. Mechanical thrombectomy with the Solitaire AB device in large artery occlusions of the anterior circulation: a pilot study. Stroke. 2010;41(8):1836-40. 
15. Brekenfeld C, Schroth G, Mordasini P, Fischer U, Mono ML, Weck A, et al. Impact of retrievable stents on acute ischemic stroke treatment. AJNR Am J Neroradiol. 2011;32(7):1269-73.

16. Davalos A, Pereira VM, Chapot R, Bonafe A, Andersson T, Gralla J. Retrospective multicenter study of Solitaire FR for revascularization in the treatment of acute ischemic stroke. Stroke. 2012;43(10):2699-705.

17. Roth C, Papanagiotou P, Behnke S, Walter S, Haass A, Becker C, et al. Stent-assisted mechanical recanalization for treatment of acute intracerebral artery occlusions. Stroke. 2010;41(11):2559-67.

18. Costalat V, Machi P, Lobotesis K, Maldonado I, Vendrell JF, Riquelme $\mathrm{C}$, et al. Rescue, combined, and stand-alone thrombectomy in the management of large vessel occlusion stroke using the solitaire device: a prospective 50-patient single-center study: timing, safety, and efficacy. Stroke. 2011;42(7):1929-35.

19. Pereira VM, Gralla J, Davalos A, Bonafé A, Castaño C, Chapot R, Liebeskind DS, Noqueira RG, Arnold M, Sztajzel R, Liebig T, Goyal M, Besselmann M, Moreno A, Schroth G. Prospective multi-centre single-arm study of mechanical thrombectomy using solitaire FR in acute ischemic stroke (STAR). Stroke. 2013;44:2802-7.

20. Saver JL, Jahan R, Levy EI, Jovin TG, Baxter B, Nogueira $\mathrm{RG}$, et al. Solitaire flow restoration device versus the Merci Retriever in patients with acute ischaemic stroke (SWIFT): a randomised, parallel-group, non-inferiority trial. Lancet. 2012;380(9849):1241-9.
21. Nogueira RG, Lutsep HL, Gupta R, Jovin TG, Albers GW, Walker GA, et al. Trevo versus Merci retrievers for thrombectomy revascularisation of large vessel occlusions in acute ischaemic stroke (TREVO 2): a randomised trial. Lancet. 2012;380(9849):1231-40.

22. Pierot L, Soderman M, Bendszus M, White P, Muto M, Turjman F, et al. Statement of ESMINT and ESNR regarding recent trials evaluating the endovascular treatment at the acute stage of ischemic stroke. Neuroradiology. 2013;55(11):1313-8.

23. von Kummer R, Gerber J. IMS-3, synthesis, and MR Rescue: no disaster, but down to earth. Clin Neuroradiol. 2013;23(1):1-3.

24. Diener HC, Foerch C, Riess H, Rother J, Schroth G, Weber R. Treatment of acute ischaemic stroke with thrombolysis or thrombectomy in patients receiving anti-thrombotic treatment. Lancet Neurol. 2013;12(7):677-88.

25. Davis MJ, Menon BK, Baghirzada LB, Campos-Herrera CR, Goyal M, Hill MD, et al. Anesthetic management and outcome in patients during endovascular therapy for acute stroke. Anesthesiology. 2012;116(2):396-405.

26. Nichols C, Carrozzella J, Yeatts S, Tomsick T, Broderick J, Khatri P. Is periprocedural sedation during acute stroke therapy associated with poorer functional outcomes? J Neurointerv Surg. 2010;2(1):67-70.

27. San Roman L, Obach V, Blasco J, Macho J, Lopez A, Urra X, et al. Single-center experience of cerebral artery thrombectomy using the TREVO device in 60 patients with acute ischemic stroke. Stroke. 2012;43(6):1657-9. 